\title{
Respuesta a gamaglobulina endovenosa en arteritis de Takayasu de difícil manejo
}

\author{
Response to intravenous gammaglobulin in \\ refractory Takayasu arteritis
}

\author{
Carlos Jaime Velásquez, Ana Paulina Brome, luisa María Benjumea, \\ Diana Carolina Cardona, Eliana Alejandra Tobón, León Darío Orrego, \\ Aura Ligia Zapata-Castellanos, Francisco IVÁn Vargas, \\ OSCAR JAíR-FELIPE-DíaZ • MEDELLÍN
}

\begin{abstract}
Resumen
La arteritis de Takayasu (AT) es una vasculitis de grandes vasos que afecta a la aorta y a sus ramas principales. Los esteroides son la piedra angular del tratamiento; sin embargo, más de la mitad de los pacientes recaen, requiriendo inmunosupresores adicionales. Estudios abiertos han sugerido que metotrexate, ciclofosfamida, azatioprina, micofenolato mofetil y terapia anti-TNF pueden ser útiles en estos casos. Se reporta un caso de una paciente con AT con múltiples recaídas pese a metotrexate, esteroides, rituximab, ciclofosfamida, infliximab, revascularización percutánea y quirúrgica. Se decidió el uso de gammaglobulina endovenosa (IVIg), con control total y sostenido de manifestaciones vasculíticas, suspensión de esteroides y disminución de los reactantes de fase aguda (Acta Med Colomb 2010; 35: 139-142).

Palabras clave: arteritis de Takayasu, gammaglobulina endovenosa.
\end{abstract}

\section{Abstract}

Takayasu arteritis (TA) is a large-vessel vasculitis which affects the aorta and its major branches. Steroids are the cornerstone of treatment; however, more than half of the patients relapse, requiring additional immunosuppressive agents. Open studies have suggested that methotrexate, cyclophosphamide, azathioprine, mycophenolate mofetil and anti-TNF therapy may be useful in such cases.

We conducted a case report of a patient with TA with multiple relapses despite methotrexate, steroids, rituximab, cyclophosphamide, infliximab, percutaneous, and surgical revascularization. Intravenous immunoglobulin (IVIg) was employed, obtaining total and sustained control of vasculitic manifestations, achieving tapering and suspension of steroids as well as diminution of acute phase reactants (Acta Med Colomb 2010; 35: 139-142).

Keywords: Takayasu arteritis; immunoglobulins, intravenous.
Dr. Carlos Jaime Velásquez Franco: Unidad de Reumatología y Enfermedades Autoinmunes, Clínica Universitaria Bolivariana. Profesor Asistente. Facultad de Medicina, Escuela de Ciencias de la Salud, Universidad Pontificia Bolivariana; Dras. Ana Paulina Brome Uribe, Luisa María Benjumea Gómez, Diana Carolina Cardona Gallón y Eliana Alejandra Tobón Palacio: Estudiantes de Medicina, Escuela de Ciencias de la Salud, Universidad Pontificia Bolivariana; Dr. León Darío Orrego: Médico, Epidemiólogo, Profesor Asistente Investigación Facultad de Medicina, Escuela de Ciencias de la Salud, Universidad Pontificia Bolivariana; Dres. Aura Ligia Zapata-Castellanos, Francisco Iván Vargas Grajales y Óscar Jaír-Felipe-Díaz: Unidad de Reumatología y Enfermedades Autoinmunes, Clínica Universitaria Bolivariana, Profesores Asistentes, Facultad de Medicina, Escuela de Ciencias de la Salud, Universidad Pontificia Bolivariana. Medellín, Colombia.

Correspondencia. Dr. Carlos Jaime Velásquez Franco.

E-mail: carjaivel@hotmail.com

Recibido: 11/XII/09 Aceptado: 18/VIII/10

\section{Introducción}

La Arteritis de Takayasu (AT) es una vasculitis de grandes vasos crónica y progresiva, que afecta la intima y adventicia de la aorta y a sus ramas principales, así como arterias pulmonares y coronarias, causando diferentes tipos de estenosis, dilataciones u oclusiones. Uno de los rasgos característicos es su predominio en las mujeres jóvenes de países de Asia y América Latina $(1,2)$.

Los esteroides son la piedra angular del tratamiento; sin embargo, más de la mitad de los pacientes recaen $(3,4)$, requiriendo inmunosupresores adicionales. Estudios abiertos han sugerido que metotrexate, ciclofosfamida, azatioprina, micofenolato mofetil y terapia anti-TNF pueden ser útiles en estos casos (5).

Hasta donde se sabe, no hay descripciones de caso del uso de gammaglobulina endovenosa (IVIg) en esta entidad. La IVIg ha sido utilizada por más de 25 años en el tratamiento de deficiencias de anticuerpos $(6,7)$. Recientes estudios han demostrado que la administración de IVIg resulta en la modulación de la respuesta inmune; además sugieren que IVIg puede tener un papel terapéutico en enfermedades autoinmunes específicas. Se presenta el caso de una paciente con AT con múltiples recaídas pese a la utilización de varios inmunosupresores que tuvo respuesta satisfactoria a IVIg. 


\section{Presentación del caso}

Mujer de 19 años con diagnóstico de arteritis de Takayasu desde 2006, por hallazgo clínico de diferencias en pulsos de extremidades superiores. Se realiza arteriografía que evidencia estenosis de aorta abdominal, oclusión de arterias: vertebral derecha, carótida derecha (Figuras 1 y 2), subclavia derecha (Figura 2) y estenosis de aorta torácica. Dentro de los paraclínicos, tenía velocidad de sedimentación globular (VSG) de $100 \mathrm{~mm} / \mathrm{h}$ y proteína C reactiva (PCR) de $1.5 \mathrm{mg} / \mathrm{dL}$. Por la severidad del compromiso vascular, requirió injerto aortosubclavio derecho. Ante estos hallazgos, se inicia tratamiento con metotrexate $20 \mathrm{mg}$ intramuscular (IM) semanales y prednisolona (PDN) $60 \mathrm{mg}$ diarios, con mejoría clínica y paraclínica sostenida por un año.

En marzo de 2007 la paciente presenta hipertensión arterial, claudicación de miembro superior izquierdo, aumento de PCR a $3.3 \mathrm{mg} / \mathrm{dL}$ y trombocitosis (525.000 plaquetas $/ \mathrm{mm}^{3}$ ). Se cambia esquema de tratamiento por rituximab 1 gramo IV cada dos semanas por un total de dos dosis en junio de 2007, con resolución de los síntomas y reducción de la dosis de PDN hasta $7.5 \mathrm{mg}$ /día. Esta adecuada evolución se mantuvo por seis meses.

En febrero de 2008 consulta por carotidinia. Se evidencia, en ecografía doppler, estenosis crítica de carótida común derecha. Se utilizaron pulsos de metilprednisolona de 500 mg diarios por tres dosis y 1 gramo IV de ciclofosfamida, angioplastia y stent de carótida derecha.

En mayo 2008 presenta reestenosis de ambas carótidas documentada por control ecográfico y por inicio de cefalea severa, carotidinia y vértigo. Se inició infliximab $200 \mathrm{mg}$ IV, presentando mejoría de los síntomas.

A las dos semanas del inicio de infliximab, la paciente consulta por dolor intenso tipo cólico en epigastrio y ambos hipocondrios. En el examen clínico se encuentra paciente con fascies cushingoides, álgida, taquicardia (frecuencia cardiaca de 110 por minuto), afebril, no se palpan pulsos femorales ni pedios, con dolor a la palpación de hemiabdomen superior sin signos de irritación peritoneal. Se hospitaliza para estudio. Se realizan paraclínicos que muestran aumento de los reactantes de fase aguda (VSG $95 \mathrm{~mm} / \mathrm{h}$ ) y leucocitosis $\left(15.600\right.$ leucocitos $\left./ \mathrm{mm}^{3}\right)$. Se realiza TAC abdominal contrastado evidenciando engrosamiento de la aorta con estenosis de la aorta inferior a la arteria mesentérica superior hasta de 50\% y disminución de la luz de la arteria mesentérica superior a nivel del ostium alrededor de un $50 \%$ y estenosis severa de aorta abdominal infrarrenal (Figuras 3 y 4 ).

Por estos hallazgos, se decide, en junio de 2008, iniciar gammaglobulina endovenosa (IVIg) $400 \mathrm{mg} / \mathrm{kg} / \mathrm{día}$ por cinco días, cada mes, por cinco meses. La paciente presenta resolución completa del dolor abdominal a los dos meses. Se logró reducción de la dosis de PDN de 50 mg a 20 mg en el tercer mes y supresión total de esteroides al sexto mes. La paciente no presentó recaídas hasta seguimiento en junio de 2009, con disminución de los reactantes de fase aguda: PCR

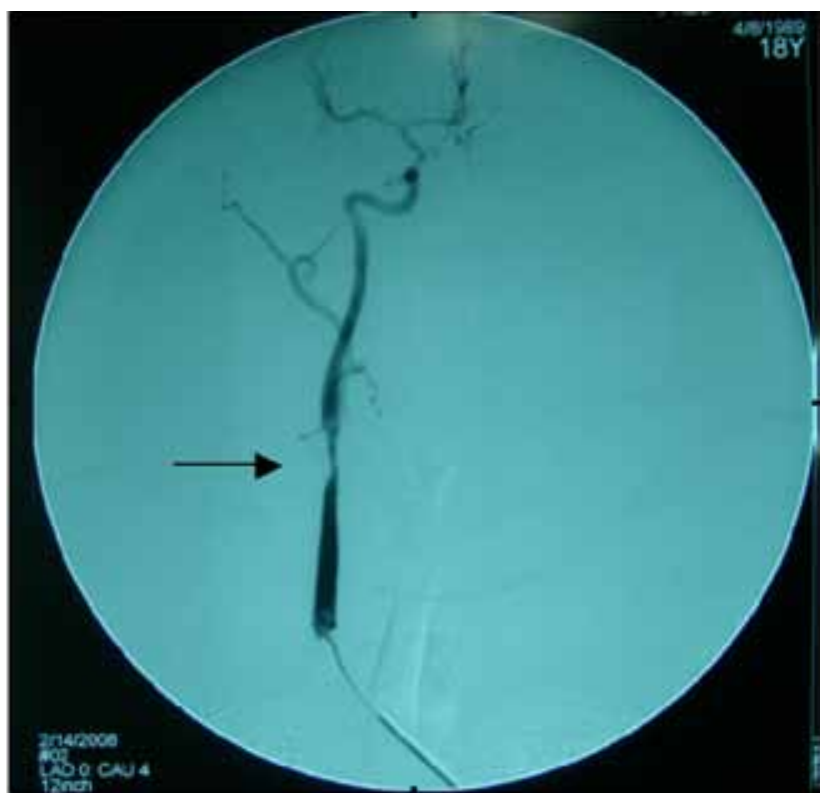

Figura 1. Angiografía digital que evidencia estenosis crítica de arteria carótida derecha (flecha).

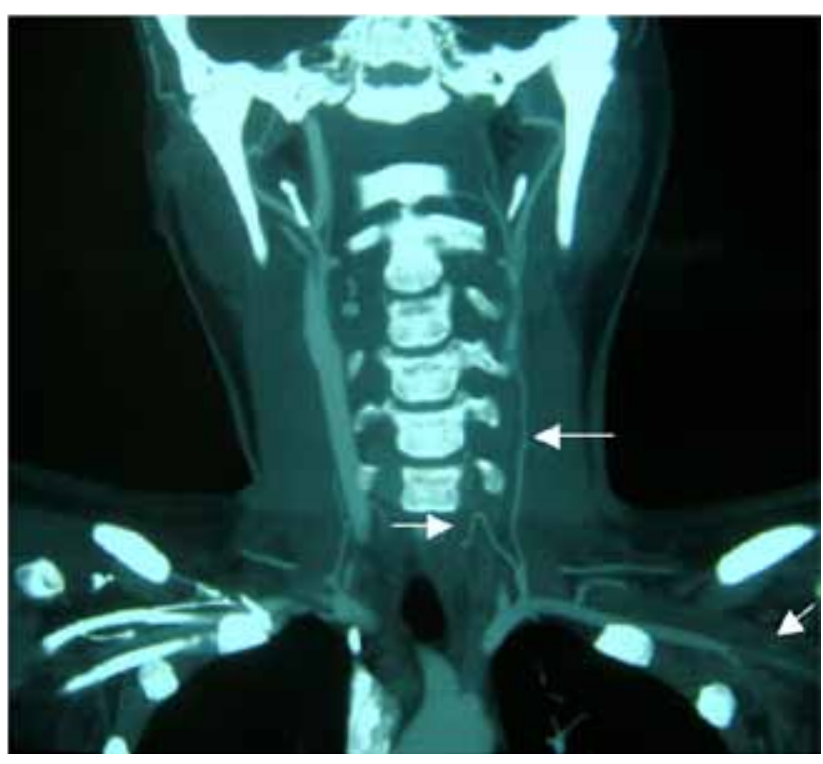

Figura 2. Angiorresonancia que evidencia estenosis severa de arterias: carótidas, vertebrales y subclavias (flechas).

que pasó de $7.8 \mathrm{mg} / \mathrm{dL}$ a $4.4 \mathrm{mg} / \mathrm{dL}$ y VSG que cambió de $108 \mathrm{~mm} / \mathrm{h}$ a $35 \mathrm{~mm} / \mathrm{h}$.

En dúplex carotídeo de 2007 se encontraba obstrucción de puente aortocarotídeo izquierdo desde su origen sin reconstitución distal; en un dúplex de control en junio de 2009 el puente subclavio-subclavio estaba permeable.

\section{Discusión}

La arteritis de Takayasu (AT) es una enfermedad inflamatoria inespecífica de origen desconocido; causa 

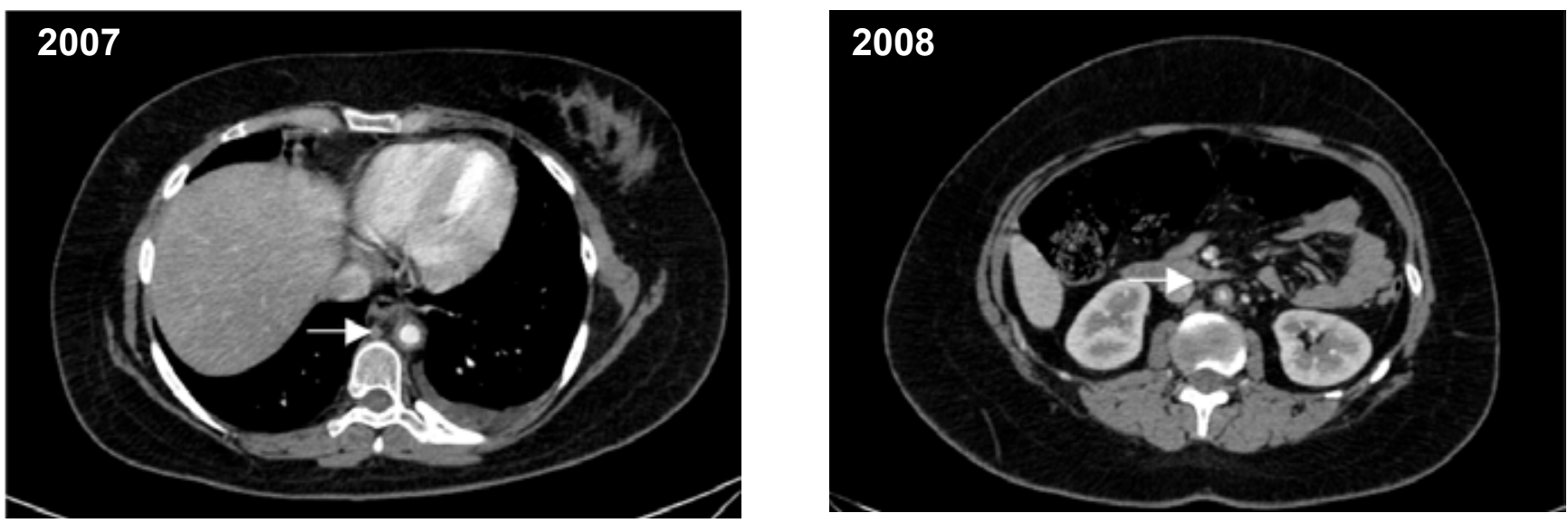

Figuras: 3 y 4. AngioTAC abdominales de 2007 y 2008 que evidencian compromiso progresivo estenótico severo de la aorta abdominal infrarrenal con marcado engrosamiento de la pared arterial y disminución de la luz (flechas).

diferentes tipos de estenosis, dilataciones u oclusiones vasculares (1).

Esta panarteritis inespecífica afecta la íntima y la adventicia de la aorta y de sus ramas (arco aórtico, aorta torácica descendente o abdominal, arterias renales, arterias coronarias, y arterias pulmonares). Sus manifestaciones clínicas son variadas y relacionadas con los vasos que presentan la lesión. En algunos casos pueden desarrollarse aneurismas e insuficiencia valvular por dilatación de la aorta ascendente $(2,3)$.

Para su diagnostico han sido ampliamente aplicados los criterios de Ishikawa y se ha clasificado en la XI Conferencia Internacional en 1994.

El tratamiento farmacológico inicial consiste en dosis altas de corticosteroides. Desafortunadamente, más del 50\% de los pacientes presenta recaídas con monoterapia con estos medicamentos (4); por esto, se han utilizado drogas citotóxicas desde el inicio de la enfermedad como terapia combinada. El uso de estos agentes ha resultado en un alto grado de control de la enfermedad, pero las recaídas tardías desafortunadamente ocurren, y aun cuando la vasculitis es bien controlada, un número significativo de pacientes experimenta efectos adversos relacionados con el tratamiento. Por estas limitaciones se han buscado terapias alternativas, incluyendo: plasmaféresis, ciclosporina A, inflixima, entre otras (5).

Recientes estudios han demostrado que la administración de IVIg resulta en la modulación de la respuesta inmune y ser una alternativa terapéutica en enfermedades autoinmunes específicas (6).

$\mathrm{Su}$ efectivo uso fue demostrado primero en púrpura trombocitopénica idiopática hace dos décadas. Desde esto se ha establecido como tratamiento benéfico alternativo en patologías tales como: Guillain-Barré, polineuropatía crónica desmielinizante, miastenia gravis, dermatomiositis refractaria, hipogammaglobulinemia, rechazo de trasplante renal, fascitis necrotizante, anemia hemolítica autoinmune y enfermedad de Kawasaki (7).

El tratamiento con IVIg para las vasculitis sistémicas ha surgido a raíz de dos hechos: 1. La habilidad de IVIg para disminuir la incidencia de aneurisma coronario en la enfermedad de Kawasaki. 2. La identificación de anticuerpos antiidiotipos reactivos con vasculitis asociadas a anticuerpos contra el citoplasma del neutrófilo (ANCAs). Existen varias presentaciones de casos del uso de IVIg en varias condiciones vasculíticas, incluyendo: crioglobulinemia, púrpura Henoch-Schönlein, poliarteritis nodosa y granulomatosis de Wegener (8).

Se han propuesto varios mecanismos de acción de la IVIg $(6,7)$ : modulación de la función del receptor Fc, protección de la superficie de membrana de la célula, depuración de agentes infecciosos, supresión de síntesis de anticuerpos, inhibición de producción y liberación de citoquinas (IL-1, TNF, IL-6), supresión de la activación de células T y superantígenos, disminución de moléculas de adhesión celular (ICAM-1, ELAM), sobrerregulación del receptor antagonista de IL-1, regulación de la función de células T y B por administración de anticuerpos antiidiotipo e inhibición de unión y activación de los componentes del complemento.

En un estudio de vasculitis sistémicas, en el que se empleó IVIg a dosis de $400 \mathrm{mg} / \mathrm{kg} /$ día por cinco días, 13 pacientes tuvieron una respuesta total y otros 13 tuvieron una respuesta parcial, respaldada por negativización de los títulos ANCAs y disminución de los reactantes de fase aguda. La eficacia se mantuvo en 18 pacientes, pero seis tuvieron recaídas que requirieron el inicio de terapia inmunosupresiva adicional. También hubo una reducción en la dosis de ciclofosfamida y prednisona en los pacientes tratados con IVIg. Particularmente nueve pacientes, previamente no tratados, recibieron IVIg como única terapia; de éstos, todos mejoraron inicialmente y cuatro tuvieron remisión completa por un año (9).

Un segundo estudio, con 15 pacientes con vasculitis asociada a ANCAs y con pobre respuesta al tratamiento convencional, consistió en la administración de 30 gramos (g) de IVIg por día por cinco días. Se presentó mejoría en seis de 15 pacientes en vasculitis limitada a un solo órgano, como piel o tracto respiratorio superior (10). 
Hasta donde se sabe, este es el primer caso publicado que demuestra la eficacia de IVIg en AT.

Existe un caso (11) de una mujer latina de 53 años con una inmunodeficiencia común variable que venía en tratamiento con IVIg desde hace tres años y que inició cuadro de dolor en brazo izquierdo, debilidad, parestesias y posterior claudicación. No se encontró presión arterial ni pulso radial en esa extremidad, disminución de pulso carotídeo izquierdo y un soplo subclavio izquierdo. La angiografía demostró obstrucción de las arterias: subclavia y vertebral izquierdas. Se aumentó la dosis de IVIg a $800 \mathrm{mg} / \mathrm{kg}$ cada tres semanas, con mejoría de los signos vitales. A los 10 meses, presenta nuevamente claudicación en miembro superior izquierdo; recibió prednisolona a $1 \mathrm{mg} / \mathrm{kg} /$ día y se aumentó IVIg a 1 $\mathrm{g} / \mathrm{kg}$ cada tres semanas, con mejoría sostenida y sin reportar ningún efecto adverso.

La decisión de utilizar este medicamento se basó en la ausencia de otras alternativas terapéuticas y por las múltiples recaídas de esta paciente pese al uso de todos los regímenes inmunosupresores e inmunomoduladores disponibles que han demostrado algún grado de efectividad en esta entidad. Se decidió apelar al efecto pleiotrópico inmunomodulador de este medicamento en todos los componentes del sistema inmune $(6,7)$ y porque ha demostrado eficacia en otras vasculitis sistémicas (8-10).

Se resalta la rápida y sostenida resolución de las manifestaciones vasculíticas, por un largo periodo de tiempo (mayor a tres meses). En estudios realizados de IVIg y granulomatosis de Wegener, comparando con grupo placebo, los pacientes lograron un beneficio con relación a la actividad de la vasculitis, la frecuencia de recaídas y la exposición a la inmunosupresión, pero éste no permaneció más allá de tres meses (11). Además se resalta el importante efecto ahorrador de esteroides hasta su suspensión total y la temprana y sostenida disminución de los reactantes de fase aguda.
En conclusión, debe considerarse el uso de IVIg en pacientes con AT con recaídas pese a la utilización de esteroides, citotóxicos y terapia biológica, con mejoría a corto plazo de las manifestaciones vasculíticas, reactantes de fase aguda y con un efecto sostenido en el ahorro de esteroides.

\section{Fuentes de ayuda}

Propias.

\section{Ninguna \\ Declaración de conflictos de interés}

\section{Referencias}

1. Watts RA, Scott DG. Epidemiology of the vasculitides. Curr Opin Rheumatol 2003; 15: 11-6.

2. Ramos LF, Pinto LF. Vasculitis de grandes vasos. Rev Colomb Reumatol 2003; 10: $19-29$

3. Ogino H, Matsuda H, Minatoya K, Sasaki H, Tanaka H, Matsumura Y et al. Overview of Late Outcome of Medical and Surgical Treatment for Takayasu Arteritis. Circulation 2008; 118: 2738-47.

4. Drigo I, Saccari A, Bacchin C, Barbi E, Bartoli F, Decorti G et al. Glucocorticoid resistance in a girl with Takayasu's arteritis. Ann Rheum Dis 2006; 65: 689-90.

5. Tanaka F, Kawakami A, Iwanaga N, Tamai M, Izumi Y, Aratake K et al. Infliximab is effective for Takayasu arteritis refractory to glucocorticoid and methotrexate. Intern Med 2006; 45: 313-6.

6. Kazatchkine MD, Kaveri SV. Immunomodulation Of Autoimmune And Inflammatory Diseases With Intravenous Immune Globulin. N Engl J Med 2001; 345: $747-55$.

7. Dalakas MC. Intravenous Inmunoglobulin in Autoinmune Neuromuscular Diseases. JAMA 2004; 291: 2367-695.

8. Klassen LW, Calabrese LH, Laxer RM. Intravenous Immunoglobulin In Rheumatic Diseases. Rheum Dis Clin North Am 1996; 22: 155-73.

9. Jayne DR, Lockwood CM. Pooled intravenous immunoglobulin in the management of systemic vasculitis. Adv Exp Med Biol 1993; 336: 469-72.

10. Jerschow E, De Vos G, Hudes G, Rubinstein A. Lipsitz EC, Rosenstreich D. A case of common variable immunodeficiency syndrome associated with Takayasu arteritis. Ann Allergy Asthma Immunol. 2007; 98: 196-9.

11. Lockwood CM. New treatment strategies for systemic vasculitis: the role of intravenous immune globulin therapy. Clin Exp Immunol 1996; 104: 77-82. 